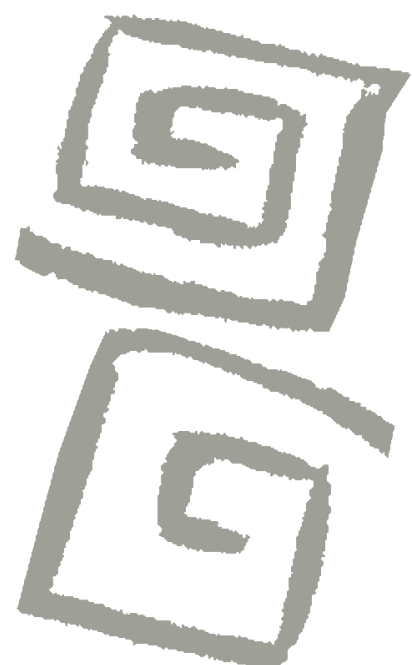

\title{
Trabajadores del sexo y salud pública: intersecciones, vulnerabilidades y resistencia
}

\author{
Sex workers and public health: intersections, \\ vulnerabilities and resistance
}

Alexandra Oliveira', Luís Fernandes ${ }^{2}$

'Doctora en Psicología. Profesora Auxiliar, Faculdade de Psicologia e de Ciências da Educação, Universidade do Porto, Portugal. $\triangle$ (iD)

${ }^{2}$ Doctor en Psicología, Profesor Asociado, Faculdade de Psicologia e de Ciências da Educação, Universidade do Porto, Portugal. $\bowtie$
RESUMEN Desde el siglo XIX, con la sífilis y, más recientemente, con el sida, Ixs trabajadorxs del sexo pasaron a ser vistos como medios de transmisión de enfermedades y como un problema de salud pública que requiere intervención. Sin embargo, las investigaciones han demostrado que, en los países occidentales, la tasa de VIH en personas involucradas con la venta de sexo es baja, con excepción de grupos específicos, como los consumidores de drogas por vía inyectable. Además, se han puesto en evidencia los riesgos a los que están sometidos Ixs trabajadorxs del sexo, por vía de la estigmatización o de otras formas de violencia. En este artículo, a partir de una etnografía urbana con trabajadorxs del sexo de calle, Ilevada a cabo en la ciudad de Porto (Portugal) entre 2004 y 2005, discutimos las vulnerabilidades sociales, laborales y jurídicas que afectan a las personas involucradas en el comercio del sexo y cómo interfieren en su salud. Nos centraremos en las estrategias de Ixs trabajadorxs del sexo para minimizar los riesgos para la salud y el discurso de resistencia en el combate a las vulnerabilidades.

PALABRAS CLAVES Trabajadores Sexuales; Salud Pública; Enfermedades de Transmisión Sexual; Gestión de Riesgos; Portugal.

\begin{abstract}
Since the 19th century with syphilis and most recently with AIDS, sex workers have been seen as a means for disease transmission and a public health problem that requires intervention. However, researchers have shown that in Western countries, HIV rates in people involved in commercial sex are low, except for in specific groups, such as intravenous drug users. Moreover, the risks faced by sex workers due to stigmatization and other forms of violence have been put into evidence. Based on an urban ethnography with street sex workers carried out in Porto (Portugal), between 2004 and 2005, this article discusses the social, labor, and legal vulnerabilities affecting people involved in commercial sex and how these interfere with their health. Focus is placed on the strategies used by sex workers to minimize health risks and their discourses of resistance in fighting vulnerabilities.
\end{abstract}

KEY WORDS Sex Workers; Public Health; Sexually Transmitted Diseases; Risk Management; Portugal. 


\section{INTRODUCCIÓN}

Las categorías que hoy circulan en el discurso tanto de cientistas sociales como de los diversos actores del debate sociopolítico, con relación a los individuos, grupos y territorios considerados problemáticos, cargan en su semántica una doble peligrosidad: la moral y la sanitaria. Esta afirmación sería fácilmente demostrada si hiciéramos la genealogía de figuras como el toxicodependiente ${ }^{(1)} \mathrm{o}$ de la periferia urbana degradada ${ }^{(2)}$. En este trabajo, elegimos una figura que convoca, desde hace tiempo, discursos y prácticas morales y sanitarias: la prostituta. Sobre esta figura situada en una zona de ambigüedad entre la normalidad y el desvío se asienta una categoría más reciente: Ixs trabajadorxs del sexo. Las expresiones trabajo sexual y trabajadores del sexo fueron propuestas en la década de 1970, por Carol Leigh, una prostituta y activista de EE.UU., como una forma de desestigmatizar y de acentuar las cuestiones laborales.

A partir de una etnografía urbana con trabajadorxs del sexo de calle, llevada a cabo en la ciudad de Porto (Portugal) ${ }^{(3)}$, discutimos las vulnerabilidades sociales, laborales y jurídicas que afectan a las personas involucradas en el comercio del sexo y cómo estas interfieren en su salud. Nos centraremos, además, en las estrategias de Ixs trabajadorxs del sexo para minimizar los riesgos de salud, y en el discurso de resistencia en el combate a las vulnerabilidades.

\section{Intersecciones entre trabajo sexual y enfermedades: construcción y deconstrucción de Ixs trabajadoxs del sexo como un problema de salud pública}

Durante el siglo XIX, cuando la sífilis alcanzó tasas preocupantes, se consideraba que las prostitutas eran uno de los principales agentes propagadores de la enfermedad. Así, con el objetivo de conocer y controlar el fenómeno, las autoridades de salud les encargaron a los médicos higienistas el estudio de la propagación de la sífilis entre las prostitutas. En Portugal, esta investigación fue dirigida por Santos Cruz, en $1841^{(4)}$, y buscó caracterizar a las prostitutas y conocer las causas de su actividad. En aquella época, los médicos higienistas -antecesores de la especialidad en salud pública- describían a las mujeres prostitutas, creyendo que de ese modo podrían percibir las causas de su actividad e impedir la diseminación del virus sifilítico. Como consecuencia de los resultados del estudio de Santos Cruz, Pina Manique, el intendente general de la Policía, ordenó las consultas médicas obligatorias y el registro policial. Nacía así la reglamentación de la prostitución cuyo objetivo explícito era la protección de la salud pública, que si bien surge a propósito de la prostituta, se extiende a otras categorías marginales (el vagabundo, el vago, el loco): el traspaso de la peligrosidad moral a la peligrosidad sanitaria, que afirman a las disciplinas científicas emergentes como los nuevos y más eficaces instrumentos de lo que Foucault denomina como sociedad disciplinaria ${ }^{(5,6)}$.

Las investigaciones continuaron a cargo de otros médicos higienistas ${ }^{(7,8)}$ y la regulación terminó recién en 1963, cuando la prostitución pasó a ser codificada como crimen $^{(9)}$. Este cambio de estatuto legal no solo no puso fin a la prostitución en Portugal sino que precarizó las condiciones de su ejercicio: las mujeres pasaron a estar en la calle y en la clandestinidad ${ }^{(10)}$.

A fines del siglo $\mathrm{XX}$, con el surgimiento del sida y el vínculo establecido entre esta enfermedad y el trabajo sexual, resurgió la voluntad de vigilancia epidemiológica y la regulación de la actividad de las personas involucradas. El miedo de que Ixs trabajadorxs del sexo pudieran transmitir la infección a sus clientes que, a su vez, infectarían a sus esposas o compañeras, trasmitiendo el VIH a la población en general, justificó las medidas de control en nombre de la salud pública y dio lugar a discursos neoreglamentaristas $y$ a un cierto pánico social. De este modo, a partir de la década de 1980, Ixs trabajadorxs del sexo pasaron a estar, de nuevo, bajo una mayor vigilancia ${ }^{(11)}$. Sin embargo, el control 
no se centró en reglamentos, sino en el estudio de las características y de los determinantes de la infección y en la implementación de proyectos de control y prevención de enfermedades sexualmente transmisibles en trabajadorxs del sexo.

Como señalan Scambler y Scambler ${ }^{(12)}$, la investigación y la intervención orientadas a la mejora de la salud de Ixs trabajadorxs del sexo surgieron, paradójicamente, a partir de que se dio a conocer la probabilidad de que el sida se transmitiera a la población heterosexual normativa, lo que generó cierto pánico moral en torno de ese hecho, y Ixs trabajadorxs del sexo pasaron a estar bajo una fuerte vigilancia epidemiológica.

Las políticas de reducción de riesgos y daños y la promoción de la salud han sido el objetivo central de la gran mayoría de los proyectos de intervención para trabajadorxs del sexo en Europa. En Portugal, el primer proyecto de intervención orientado a los riesgos de contraer enfermedades como el $\mathrm{VIH} /$ sida y otras infecciones de transmisión sexual, entre trabajadoras del sexo y que incluía una línea de reducción de riesgos asociados al trabajo sexual, el programa Drop In, surgió en 1994, en el barrio Intendente, en Lisboa, inserto en un centro de salud ${ }^{(12,13)}$.

Luego surgieron otros proyectos de intervención (VAMP, Autoestima, Espaço Pessoa, Red Light, Adão e Eva, etc.), sobre todo por iniciativa de la sociedad civil -organizaciones no gubernamentales (ONG)-, pero también de servicios de salud estatales financiados, en su mayoría, por el programa nacional de lucha contra el sida. Un relevamiento efectuado en $2014^{(14)}$ mostró la existencia 14 ONG que desarrollaban intervenciones de reducción de riesgos en el área de trabajo sexual, y de 28 proyectos activos con 82 técnicos para intervención directa con trabajadorxs del sexo. La reducción de riesgos tiene marco legal en Portugal desde 2001, cuando surgió el Decreto Ley 183/2001 del 21 de junio, que aprobó el régimen general de las políticas de minimización de daños y condujo al surgimiento de propuestas y estrategias de intervención basadas en los principios del humanismo y del pragmatismo ${ }^{(15)}$.
A pesar de todas estas intervenciones, las investigaciones comenzaron a mostrar que la tasa de VIH en trabajadorxs del sexo es baja, excepto en grupos específicos, como los consumidores de drogas por vía inyectable o los hombres que tienen sexo con hombres ${ }^{(16)}$. En este sentido, Vanwesenbeeck ${ }^{(17)}$, a partir de una revisión bibliográfica, presenta resultados de estudios de prevalencia del $\mathrm{VIH}$, tanto en Europa, como en EE.UU., que indican que Ixs trabajadorxs del sexo que están infectadxs son principalmente consumidorxs de drogas por vía inyectable.

Otro dato pertinente para la deconstrucción de la asociación entre trabajo sexual e infecciones sexualmente transmisibles es el conocimiento de que, en las sociedades occidentales, Ixs trabajadorxs del sexo reportan tasas de utilización de preservativo elevadas con clientes y bajas con compañeros o amantes ${ }^{(18,19,20,21)}$, por lo que en los contextos no profesionales puede existir un mayor riesgo de contraer la enfermedad ${ }^{(17)}$.

Estas elevadas tasas de utilización del preservativo contrastan con el gran número de propuestas de sexo desprotegido que Ixs trabajadorxs del sexo dicen recibir de sus clientes o potenciales clientes. De acuerdo con las respuestas obtenidas en una encuesta realizada con profesionales del sexo que trabajan en pisos de contacto $^{(20)}$, es muy frecuente que reciban propuestas de sexo no seguro por parte de sus clientes. Al preguntar a Ixs participantes de esa investigación qué hacían en esas situaciones, todxs respondieron que se negaban a ceder, pues no querían estar en riesgo. Estos datos son consistentes con los de otra investigación que llevamos a cabo en la ciudad de Porto ${ }^{(3)}$, en la que Ixs trabajadorxs del sexo afirmaban tener una actitud pedagógica con sus clientes acerca de cuestiones relacionadas con la salud, las enfermedades y los peligros del sexo no protegido. Por esta razón, algunos autores $^{(22,23)}$ han acentuado el papel de Ixs trabajadorxs del sexo como educadores sexuales, convirtiéndolos en aliados en la lucha contra el $\mathrm{VIH} /$ sida y otras infecciones de transmisión sexual y en la educación para la salud, lo que obliga a confrontar la 
creencia socialmente dominante de que son vehículos de la enfermedad. De este modo, cuando se analiza la relación entre trabajo sexual y enfermedades no se puede olvidar a los clientes y la baja percepción del riesgo que muestran al intentar tener sexo sin protección, poniendo en riesgo la salud de las personas que ejercen sexo comercial.

Aun más, se han puesto en evidencia los riesgos a los que están sometidos Ixs trabajadorxs del sexo a través de la estigmatización y de otras formas de violencia. La reacción social ante las personas que hacen trabajo sexual fue, y continúa siendo, de rechazo y de exclusión. La estigmatización, la discriminación, la exclusión y las diversas formas de violencia son las expresiones más visibles y nefastas de una reacción social negativa ante las personas que ejercen el trabajo sexual. Además, la elevada tasa de víctimas entre Ixs trabajadorxs del sexo, en las más diversas formas y en los diferentes contextos del trabajo sexual, ha sido ampliamente demostrada ${ }^{(24,25,26)}$.

En suma, podemos concluir que, a pesar de la histórica asociación entre trabajo sexual y ciertas enfermedades, como la sífilis y el sida, más que constituir un problema de salud pública, Ixs trabajadorxs del sexo están en riesgo, tanto por los intentos de sexo sin protección por parte de los clientes, como por el estigma y otras formas de violencia.

\section{ASPECTOS METODOLÓGICOS: ETNOGRAFÍA DEL TRABAJO SEXUAL DE CALLE EN PORTO}

Sobre la base del método etnográfico, realizamos una investigación en el centro de Porto $^{(3)}$ que nos permitió conocer el mundo social del trabajo sexual de calle en un contexto urbano $y$, en particular, a Ixs trabajadorxs del sexo que lo habitan. Nos adentramos en el trabajo de campo con la finalidad de caracterizar, comprender e interpretar diversos aspectos del mundo social del trabajo sexual de calle y de los actores que lo componen.
Los objetivos se centraron en el conocimiento de las trayectorias de vida de las personas que hacen trabajo sexual, los procesos subyacentes a la iniciación en esta actividad y los significados que les atribuyen; los clientes del trabajo sexual y los contextos de estas actividades, como también el propio ejercicio de la venta de sexo y las interacciones que se producen entre los diversos actores; y la reacción social frente a los actores y actos del sexo comercial de calle, tanto por parte de individuos o grupos, como por parte de las instituciones.

La elección de la etnografía se basó en la voluntad de acceder a una posición perceptiva más cercana, una de las características que más distingue al método etnográfico de otras estrategias empíricas, sobre todo cuando están en juego fenómenos de estigma o de juicio moral condenatorio. La observación, en sus diferentes modalidades, incluyendo la observación participante y el contacto con informantes claves fueron las principales técnicas utilizadas para la colecta de datos. Seguimos así el principio fenomenológico que toma la experiencia personal como fuente de conocimiento, que se torna más relevante aún cuando se trata de obtener un conocimiento preciso sobre comportamientos considerados desviantes.

La etnografía se caracteriza por tener como principal instrumento de investigación al propio investigador y como procedimientos centrales la presencia prolongada en el contexto en estudio y el contacto directo con las personas, las situaciones y los acontecimientos $^{(27)}$. Si bien el alcance temporal de la observación fue bastante extenso y abarcó cinco años, la observación participante se desarrolló en forma intensiva y sistemática entre octubre de 2004 y octubre de 2005. Nuestra permanencia en terreno fue, en promedio, de tres días por semana y de entre 15 minutos y 12 horas, sumando casi 400 horas solo en los años de 2004 y 2005. Durante todo este período de tiempo, nos concentramos en ver qué hacían las personas, oír qué decían y experimentar las circunstancias por las que atravesaban sus vidas $^{(28)}$. 
En diversos lugares de trabajo sexual de calle, en la zona central de la ciudad de Porto, formamos parte de todas las actividades diarias y rutinarias, excepto el intercambio de sexo por dinero. Esto implicó permanecer en la calle con Ixs trabajadorxs del sexo, esperar que los clientes se acerquen, observar y participar de la interacción que entablaban con ellos, conversar con las dueñas y las empleadas de los lugares en que se concretaban las relaciones sexuales pagas, sobre todo cuando todxs Ixs trabajadorxs del sexo estaban ocupadxs; pero también conocer a sus hijos, sus compañeros y otros familiares, comer con ellas y participar de sus actividades recreativas.

En el terreno, la observación participante se realizó en todos aquellos lugares a los que nos llevó el hecho de involucrarnos con los actores. Interactuábamos siempre y cuando las mujeres y las transgénero lo permitieran, propusieran o cuando sucedía inesperadamente. La observación se efectuó en variados contextos y con diversos actores, pero los lugares de observación y de interacción fueron casi siempre la calle, y los protagonistas centrales eran Ixs trabajadorxes sexuales.

Aunque la propuesta era abarcar hombres, mujeres y transgénero, entre las personas que practican el trabajo sexual de calle, las mujeres cisgénero fueron con las que mejor conseguimos alcanzar los objetivos. La aceptación que obtuvimos en algunos lugares con algunas mujeres cis fue total, mientras que en otros lugares tanto con mujeres cis o trans, la aceptación fue solo parcial.

Además de la observación participante, en la que participaron 108 mujeres, hombres y transgéneros y a quienes entrevistamos de manera informal, realizamos 32 entrevistas en profundidad a 28 mujeres cis y cuatro mujeres trans trabajadoras sexuales. De estas, 23 eran portuguesas y nueve extranjeras (seis brasileñas, dos rumanas y una española) y sus edades variaban entre los 21 y los 60 años. En este grupo, había tres toxicodependientes, dos ex toxicodependientes y dos con problemas de alcoholismo.
Todxs Ixs participantxs fueron informados previamente de los objetivos de la investigación, así como del carácter voluntario de su participación y del anonimato, y se obtuvo el consentimiento informado oral o tácito. En lo que concierne a esta cuestión, consideramos que no hay mejor evidencia del consentimiento informado que aquel que se obtiene al ganar el acceso y la aceptación como investigador y se conquista la colaboración de participantes como aquellos que conforman el mundo del trabajo sexual de calle (o los de otros contextos similares).

Esta investigación, en la que habitamos el mundo de la prostitución de calle de forma prolongada, profunda e intensa, nos permitió acceder al punto de vista de sus actores: cómo se ven, los significados que atribuyen a su vida, cómo viven los mundos que ocupan, cómo se sitúan en relación con los demás y cómo se posicionan con relación a las reglas y a la sociedad dominante. Nos brindó la posibilidad de tener una percepción más próxima de su mundo social: cómo se organiza, qué territorios ocupa, cuáles son sus dinámicas y qué tipo de relaciones lo componen. Haber permanecido allí nos permitió, además, experimentar muchas de las reacciones sociales negativas con relación a ese mundo por parte de aquellos que no son de allí.

Así, reunimos un conjunto de conocimientos que incluyen las diversas vulnerabilidades que alcanzan a Ixs trabajadorxs del sexo y de las cuales daremos cuenta a continuación. Luego de esta etnografía, realizamos otros trabajos de investigación con personas que prestan servicios sexuales pagos, lo que nos ha permitido consolidar y corroborar los resultados de esta investigación etnográfica. Entre las investigaciones que la primera autora de este texto ha dirigido y que contribuyen al fortalecimiento de los conocimientos con este objeto, destacamos una investigación realizada en Lisboa con trabajadorxs del sexo de pisos de contacto $^{(20)}$, una investigación-acción con educadores de pares trabajadorxs del $\operatorname{sexo}^{(29,30)}$ y un estudio con migrantes trabajadorxs sexuales y salud ${ }^{(31,32)}$. 


\section{VULNERABILIDADES SOCIALES, LABORALES Y JURÍDICAS ASOCIADAS AL TRABAJO SEXUAL}

A pesar de la deconstrucción de la asociación entre trabajo sexual y enfermedad, parece existir un conjunto de vulnerabilidades que pueden justificar un mayor riesgo, principalmente, de salud en Ixs trabajadorxs del sexo. Así, analizaremos su vulnerabilidad social y, posteriormente, las vulnerabilidades laborales y jurídicas.

\section{Vulnerabilidad social}

La vulnerabilidad social se puede relacionar con el estigma y, como consecuencia de este, con la victimización. Las agresiones sobre Ixs trabajadorxs del sexo de calle asumen diversas formas y grados $y$, a pesar de que muchas de las mujeres, hombres y transexuales no piden ayuda, en particular, a los servicios de salud y/o de justicia, estas acciones imprimen un elevado grado de sufrimiento en la víctima. Las violencias ocurren con gran frecuencia, afectan de forma traumática el bienestar físico y mental y pueden tener como consecuencia el tratamiento hospitalario o, incluso, resultar en homicidio.

Durante todo el trabajo de campo pudimos constatar diversos casos de agresiones físicas, sexuales y psicológicas, de mayor o menor gravedad, dirigidas a una sola persona o a un grupo y dirigidas a mujeres, hombres y transgénero. El siguiente extracto del diario de campo contiene, como ejemplo, la descripción de una agresión, con serias consecuencias, contada por la trabajadora del sexo que la sufrió:

I. fue atacada por un cliente que ya la había buscado cuatro veces. [...] miró por encima del hombro y lo vio sacar del bolso un lazo con el cual supuestamente pretendía estrangularla, pues lo pasó alrededor de su cuello. Ella intentó escapar del cuarto pero, al agarrar el picaporte de la puerta que estaba cerrada, la llave se cayó. Lucharon y ella intentó salir por la ventana, pero no lo consiguió. Entonces él sacó del bolso un frasco con amoníaco y se lo lanzó en la cara, en la zona de los ojos. [...] Perdió gran parte del campo visual del ojo izquierdo y, como en el ojo derecho tenía previamente un estrabismo incorregible, quedó con una gran deficiencia visual. A pesar del uso de anteojos con mucha graduación no consigue compensar ese déficit. Nunca consiguió reconocer al individuo. (Diario de campo, 30.03.2005)

La violencia presente en el trabajo sexual de calle es abierta, dirigida, orientada, muy frecuente, con consecuencias físicas y/o psicológicas y es practicada por clientes, hombres que se hacen pasar por clientes, y otros hombres o mujeres. Nos referimos a una diversidad de actos intencionales que incluyen insultos, agresiones físicas, robos, raptos y violaciones. Las agresiones físicas directas abarcan golpes, puntapiés, amenazas con armas y arrojar objetos.

Anteayer a la noche, un auto con tres jóvenes paró en la puerta de la pensión y allí estaba solo M. Preguntaron el precio y luego comenzaron a burlarse y a insultarla, hasta la llamaron puta con toda la violencia. Cuando M. no aguantó más devolvió el insulto y dijo: "Puta será tu madre". Uno de los jóvenes se bajó del auto y ella se refugió en la pensión. M. [que terminó siendo agredida] está muy pisoteada y tiene dolores fuertes en una rodilla. (Diario de campo, 16.07.2005)

El secuestro se da con cierta frecuencia $y$, dependiendo de las circunstancias, puede tener consecuencias psicológicas severas. Varias mujeres contaron que fueron raptadas y dejadas en lugares aislados y distantes del sitio donde fueron levantadas. En esos casos, cuando son agredidas física o sexualmente y luego abandonadas sin ropa, la experiencia puede ser profundamente traumática.

Más allá de que cada unx de ellxs puede ser víctima en forma individual, Ixs 
trabajadorxs del sexo de calle son víctimas como grupo: hablamos de la violencia a la que están sujetos por el simple hecho de ser lo que son. Son víctimas colectivas, tal como se ha defendido en un trabajo anterior ${ }^{(33)}$ en el que planteamos un paralelismo entre las víctimas concebidas a partir de lo individual y lo psicológico y las que son víctimas por su pertenencia a determinados grupos, etnias o territorios. El trauma es aquí resultante de la intensificación del estigma, de la acentuación del aislamiento del grupo y de la desconfianza hacia lo externo. Ellxs son víctimas colectivas de acciones de individuos o grupos que parecen hacerlo por un rechazo del otro, como si no hubiera formas tolerables de existencia social más allá de las que se identifican con la norma moral dominante.

Esta aserción se basa en una mayor violencia hacia Ixs trabajadorxs del sexo que se caracterizan por otros comportamientos estigmatizados. Nos referimos, por ejemplo, a los casos de hombres y transexuales que hacen trabajo sexual. En estos grupos, al estigma del sexo por dinero se suma el estigma de ser homosexual, hombre en un cuerpo de mujer o travestido. Las agresiones verbales se acentuaban en estos casos y los relatos de agresiones eran muy serios. Una transexual que conocimos fue una vez engañada por un grupo de hombres que la sometió a una violación colectiva. Este acto tuvo serias consecuencias para su salud física y psicológica, y ella tuvo que recibir tratamiento hospitalario por las heridas causadas por la intensa violación que sufrió. Esta mujer trans comenzó a tener miedo a salir sola, a evitar determinados lugares y dejó de andar travestida para intentar prevenir un segundo ataque. Esta agresión fue la causa de su infección por el VIH, que causó su muerte, algunos años después.

Además de las formas de violencia que se ejercen en las calles o en los cuartos de las pensiones, constatamos formas de violencia institucional, que es aquella que surge cuando Ixs trabajadorxs del sexo tienen contacto con las instituciones estatales en las áreas de seguridad social, de salud y de justicia, y son discriminadxs y tratadxs con preconcepto. En los hospitales o centros de salud pueden sentirse estigmatizadxs y, particularmente, los travestis y transexuales son con frecuencia blanco de agresiones, sobre todo, verbales; en la justicia sus reclamos son subvalorados y no tienen seguimiento, sufren los efectos directos del desprestigio; $y$, como madres, puede ser cuestionado el derecho a la tutela de sus hijos. Vease el siguiente ejemplo:

Hoy R. me contó que fue a la Seguridad Social [...] para intentar obtener el apoyo económico que necesitaba [...]. Le contó a la asistente social su situación actual: está embarazada, vive con un hijo menor, una hija y dos nietos en un monoambiente, y tiene un pequeño salario. Le dijo además que fue prostituta. No le dijo nada más, o sea, la técnica no sabía nada más de su historia. Aún así, le preguntó por qué no daba su hijo en adopción. R. se sintió muy molesta y abrumada con esto: "Ella no me conoce de ningún lado, no sabe nada de mi vida, no sabe si soy o no buena madre, no quiso saber si alguna vez les faltó algo a mis hijos, no tenía nada que decir, fue solo porque le dije que andaba en la vida [...] No es admisible que diga una cosa así. Que ella haga hijos y que los dé, si es capaz... (Diario de campo, 18.06.2005)

Cuando se produce este tipo de violencia se refuerza el estigma y eso sucede porque las instituciones, en la figura de las personas que las representan, pueden considerar que la violencia ejercida sobre aquellas personas en las que identifican una transgresión moral es legítima. Esto sucede porque la visión estereotipada que la sociedad dominante tiene sobre las personas que se prostituyen es deshumanizada. $\mathrm{O}$ mejor dicho, las deshumaniza, y alguien que no es humano no tiene sentimientos, emociones o sensaciones y, por lo tanto, la violencia está justificada. 


\section{Vulnerabilidades laborales y jurídicas}

La vulnerabilidad laboral deriva de la propia naturaleza del trabajo y de las condiciones en las que es ejercido. El hecho de tener múltiples parejas y de desarrollar su trabajo en determinadas condiciones ecosociales puede explicar los elevados índices de violencia sobre Ixs trabajadorxs del sexo de calle. Las zonas desiertas y oscuras, frecuentadas durante la noche, con obstáculos a la visibilidad, como cobertizos y columnas, las débiles condiciones de autoprotección, la violencia sistémica de algunos de los territorios de la prostitución, la variedad de clientes y la imprevisibilidad de sus comportamientos pueden ser considerados factores de riesgo de violencia ${ }^{(34)}$. En otros casos, no controlar el lugar de encuentro y tener relaciones sexuales en el auto del cliente pueden ser indicados como predictores de la victimización ${ }^{(35)}$. Algunos autores ${ }^{(36)}$ consideran que la prostitución de calle es, sin duda, una de las ocupaciones más peligrosas en las cuales una mujer puede estar involucrada, sobre todo, porque las agresiones son fácilmente racionalizadas por los perpetradores y porque Ixs trabajadorxs del sexo no cuentan con protección social y tienen poco o ningún acceso a las instituciones de apoyo, como la policía, lo que está de acuerdo con lo que observamos en el terreno.

Esta vulnerabilidad en sus contextos de trabajo se relaciona también con una vulnerabilidad jurídica derivada de la ausencia de reconocimiento legal de la actividad de sexo comercial por parte del Estado. Al no ser una actividad reconocida como profesión no están garantizados los derechos laborales, como el derecho a vacaciones pagas y la licencia por enfermedad. El hecho de que el Estado no reconozca el trabajo sexual como una actividad profesional legítima (por ejemplo, no cobra impuestos, no regula), no impide que esté organizado como una actividad laboral y sea entendido y practicado como un trabajo. La mayoría de Ixs trabajadorxs del sexo de nuestra investigación, al practicarlo como un trabajo, defiende la idea de que legalizar o reglamentar la prostitución sería un paso relevante y necesario para el reconocimiento de su práctica, que les otorgaría los derechos, el respeto y la dignidad que están ausentes, y ayudaría a desestigmatizar y disminuir la violencia.

Al preguntar cuál sería el mejor sistema legal para la prostitución, la mayoría de Ixs trabajadorxs del sexo de calle con quienes hicimos nuestra etnografía defiende que el sistema ideal de legalización o reglamentación debía resguardar a las mujeres de la exposición pública, de las agresiones y de la intemperie, o sea, no sería compatible con la prostitución de calle. Sería un sistema que permitiría la inscripción en la seguridad social y el pago de impuestos con una declaración de ganancias anual como trabajador independiente. Dadas las características de la actividad, deberían tener consultas médicas disponibles, pero no obligatorias.

Además de todos los aspectos prácticos referidos, en particular los que están relacionados con la protección personal, lo que nos mostró el contacto cercano con muchas trabajadorxs del sexo fue que lo que buscan es el respeto y la dignidad que la sociedad siempre les negó. Y el reconocimiento de su actividad como un trabajo legal podría contribuir a eso, como refirió Z.: "Creo que soy una mujer como las otras, soy igual, ¿no lo soy? Yo creo que sí [...] ya que no pueden terminar con la prostitución, que nos traten con un poco de dignidad".

\section{Estrategias espontáneas de reducción de riesgos}

Hasta ahora vimos las diversas vulnerabilidades, los riesgos y la ausencia de reconocimiento legal. Estas condiciones podrían producir una especie de resignación que debilite toda la capacidad de reacción por parte de Ixs trabajadorxs del sexo de calle. Pero no es lo que muestran los datos: a pesar de las frecuentes agresiones no existe indiferencia, sobre todo en las situaciones más graves. $\mathrm{Si}$ alguien las insulta, se produce una reacción inmediata de devolución del insulto, pero si una mujer va al hospital porque alguien pasó 
y le tiró el polvo químico del matafuego en la cara hay indignación y revuelta. Es como si les hubiesen tirado a todxs, en realidad lo hicieron, porque lo que motivó la agresión a la colega es una característica que también es suya, es la pertenencia al grupo. Se identifican con el grupo y por eso sienten las consecuencias de esa pertenencia.

Además Ixs trabajadorxs del sexo emprenden estrategias para minimizar los riesgos a los que están sujetxs y para resistir a las vulnerabilidades, como sucede cuando un tercero cuestiona su derecho a la maternidad y tratan de prevenir esa situación. Así, una de las trabajadoras del sexo que conocimos, cuando estaba embarazada, trató de asegurarse de que, luego que su hijo naciera, la guarda quedara a cargo de su madre, por miedo a perder su tutela.

Llegó D., la joven que estaba embarazada. Le pregunté por el bebé y me respondió que estaba todo bien, que estaba con su madre. El único miedo que tenía era que la Seguridad Social se lo sacara, pero como enseguida lo llevó a la casa de su madre, no tuvo problemas. (Diario de campo, 29.04.2005)

La violencia institucional bajo la forma de discriminación todavía ocurre en las instituciones de salud. En general, si un trabajador del sexo quiere ser bien tratado y atendido en centros de salud y hospitales, la mejor opción es no mencionar su actividad. Muchas mujeres contaban historias de mala atención en los centros de salud y hospitales, por lo que preferían servicios de salud privados o servicios especializados en trabajadores del sexo como los proporcionados por los proyectos de reducción de riesgos. Además, muchxs optan por no decirle al profesional de salud cuál es su ocupación. En la investigación que llevamos a cabo en Lisboa con personas que ejercen el trabajo sexual en pisos de contacto ${ }^{(20)}$ preguntamos a nuestros participantes si al contactar a un profesional de salud mencionaban que hacían trabajo sexual en el caso de que esa información sea relevante para el problema por el que recurrió al servicio de salud. El 63,2\% respondió que nunca o casi nunca menciona que practica sexo comercial, y el $36,7 \%$ respondió que lo hace siempre o casi siempre. Así, esconder su actividad surge como una forma de evitar el estigma y la discriminación.

El estigma del trabajo sexual es disimulable ${ }^{(37)}$. Cuando una persona detenta un estigma que puede ser ocultado o mantenido en secreto, como en el caso de Ixs trabajadorxs del sexo o de lxs homosexualxs, puede poner en práctica estrategias de control de la información relacionada con la actividad o el trazo estigmatizado. Así, las personas esconden sus actividades para protegerse a sí mismas de la tensión causada por el estigma. Sin embargo, intentar controlar lo que quieren exhibir o contar, fingir o mentir y, en cada caso, a quién, cómo y cuándo, requiere un enorme esfuerzo psicológico. El intento de influir sobre lo que es revelado o no sobre sí mismos en la interacción social, exige un gran control mental ${ }^{(38)}$. Como afirman Koken, Bimbi, Parsons y Halkitis, un estigma disimulado potencia secuelas negativas más graves sobre la salud física y mental de los individuos que las provocadas por los estigmas visibles ${ }^{(39)}$. Estos autores explican cómo los profesionales del sexo pueden negarse a recibir apoyo social, con el objetivo de protegerse de la pérdida de su estatuto ante la divulgación de su actividad. La invisibilidad es, así, una estrategia de gestión del estigma y una forma de evitar el rechazo social. Asumir públicamente el sexo comercial puede llevar al desprestigio, entonces, se mantiene una simulación que causa estrés y ansiedad, como resultado de la mentira y de una doble vida.

En el caso de Ixs trabajadorxs del sexo migrantes es aún más frecuente oír relatos de tratos discriminatorios, tales como rechazar atenderlos, insultos, humillaciones y negligencia, lo que los lleva a dejar de asistir a los servicios de salud estatales. Esto los obliga a procurar cuidados de salud no oficiales y no profesionales. Una trabajadora del sexo migrante que entrevistamos ${ }^{(32)}$ relataba una experiencia negativa con una médica ginecóloga en un centro de salud. Según esta 
mujer brasileña, la médica se colocó dos pares de guantes antes de examinarla, le pide a la enfermera que tuviera más cuidado de lo habitual, la mandó a ella a sacar la protección de la camilla al finalizar el examen, le dijo que no creía que haya tenido relaciones sexuales solo con un hombre y mintió con respecto a la información que provee el Papanicolaou diciendo que a través de ese examen se podía saber con cuántos hombres había tenido relaciones sexuales. Esta mujer nunca más fue al centro de salud.

Además de estas estrategias, es habitual que Ixs trabajadorxs del sexo de calle hagan planes de protección contra las agresiones. Nos referimos a las estrategias de gestión del riesgo de ser agredidx. Estas estrategias son conocidas e implementadas por todxs: tanto mujeres, hombres y transgéneros que están en la venta de sexo de calle utilizan técnicas de protección personal para prevenir ser víctimas. En el trabajo etnográfico que presentamos aquí surgieron una diversidad de estrategias, tales como evitar lugares oscuros y aislados, esconder el dinero, no estar solxs en la calle, no llevar cartera, trabajar siempre en el mismo sitio, no entrar en los automóviles de los clientes ni de los desconocidos o cuyo aspecto físico les inspire alguna desconfianza, pedir el pago en forma anticipada, no salir del centro de la ciudad, no ir con individuos alcoholizados o drogados, entre otras. Además, algunas de las mujeres Ilevan consigo sprays para defensa personal:

Le hablé de los sprays de autodefensa y M. me mostró uno que tiene en la cartera. Dice que anda siempre con él porque es peligroso cuando sale de ahí, incluso en taxi. Dice que las personas pueden vigilarla y atacarla por pensar que Ileva mucho dinero. Tenía cierto recelo de mostrarlo porque sabe que su uso está prohibido en Portugal. El que tiene, se lo trajeron de Francia. (Diario de campo, 11.05.2005)

\section{NOTAS FINALES: RESISTENCIA A LA MARGINALIZACIÓN}

Durante nuestro trabajo de terreno pudimos verificar la existencia de actos que marcan formas de resistir a la exclusión y a la marginalización. A partir de las conversaciones informales y de las entrevistas efectuadas, con apoyo de la observación participante, constatamos que, al contrario del estereotipo defendido, Ixs prostitutxs de calle, en general, tienen un sentido político de sí, es decir, se piensan a partir de principios que defienden y de objetivos que los orientan en las opciones de vida que toman. Como tal, fuimos notando cómo se posicionaban afirmativamente con relación a su actividad y cómo lamentaban la estigmatización y la ausencia de derechos cívicos. Algunxs iban más lejos, al mencionar que un movimiento de trabajadorxs del sexo sería una contribución importante para luchar contra las causas que sustentan la discriminación a la que están sujetxs. Sin embargo, la mayoría revelaba una falta de consciencia política.

Así, constatamos que Ixs trabajadorxs del sexo lamentaban la estigmatización y la falta de derechos y reconocían la importancia de luchar por estos. A pesar de que no se haya formado una asociación de trabajadorxs del sexo en Portugal, pudimos identificar algunas acciones individuales o colectivas que pueden ser vistas como puntos de resistencia a la falta de reconocimiento social y legal. Como ejemplo, durante el período de tiempo en el que se desarrolló nuestra investigación, dos prostitutas, impulsadas por nosotras, fueron a una conferencia en Bruselas sobre trabajo sexual, derechos humanos e inmigración. Esta conferencia, organizada por y para trabajadorxs del sexo, permitió que estas mujeres contactaran con movimientos y activistas extranjeros, lo que impulsó la organización de un encuentro en Portugal. Después de la conferencia, a pretexto del Día Internacional contra la Violencia sobre Trabajadores del Sexo, algunxs trabajadorxs del sexo, con nuestra ayuda, organizaron un encuentro nacional que contó con cerca de 
30 trabajadorxs del sexo y de otros aliados a su causa que discutieron tópicos como la violencia y la necesidad de crear un movimiento en Portugal. Esta iniciativa consiguió promover en la sociedad portuguesa el debate sobre los derechos de los trabajadores del sexo, sobre todo, en los medios de comunicación social, al interior de los proyectos de intervención e incluso en las asociaciones sindicales. Consideramos que este es un buen ejemplo de la reacción y lucha contra la falta de reconocimiento social y legal al que han sido sometidxs.

\section{REFERENCIAS BIBLIOGRÁFICAS}

1. Fernandes L. Fenómeno droga e perturbação da estabilidade normativa. In: Cunha $M$, (ed.). Do crime e do castigo: temas e debates contemporâneos. Lisboa: Mundos Sociais; 2015.

2. Fernandes L, Mata S. Viver nas "periferias desqualificadas": do que diz a literatura às perceções de interventores comunitários. Ponto Urbe [Internet]. 2015 Jul 31 [cited 01 Oct 2016]. Available from: http://pontourbe.revues.org/2658

3. Oliveira A. Andar na vida: prostituição de rua e reacção social. Coimbra: Almedina; 2011.

4. Cruz FS. Da prostituição na cidade de Lisboa. Lisboa: Typ Lisbonense; 1841.

5. Foucault M. Vigiar e punir: Nascimento da prisão. 10a ed. Petrópolis: Vozes; 1993.

6. Foucault M. História de sexualidade: A vontade de saber. 10a ed. Rio de Janeiro: Graal; 1988.

7. D'Azevedo FP. Historia da prostituição e policia sanitaria no Porto. Porto: Casa de F Gomes da Fonseca; 1864.

8. Fonseca A. Da prostituição em Portugal. Porto: Typographia Occidental; 1902.

9. Oliveira A. História jurídico-legislativa da prostituição em Portugal. Revista do Ministério Público. 2004;98:145-156.

10. Oliveira A. As vendedoras de ilusões: Estudo sobre prostituição, alterne e strip tease. Lisboa: Editorial Notícias; 2004.

11. Scambler G, Scambler A. Social change and health promotion among women sex workers in London. Health Promotion International. 1995;10(1):17-24.
12. Oliveira A. O mundo da prostituição de rua: trajectórias, discursos e práticas, um estudo etnográfico. Porto: University of Porto; 2008.

13. Mak R. Health care for sex workers in Europe. In: Day S, Ward H, (eds.). Sex work, mobility and health in Europe. London: Kegan Paul; 2004. p. 123-159.

14. Coutinho J, Oliveira, A. Minimização de danos no trabalho sexual em Portugal: As representações dos técnicos interventores. Psicologia, Saúde e Doenças. 2014;15(2): 538-553.

15. Fernandes L, Ribeiro C. Minimização de danos, estilos de vida junkie e controlo social. Sociologia, Problemas e Práticas. 2002;39:57-68.

16. Gaffney J, Velcevsky P, Phoenix J, Schiffer K. Practical guidelines for delivering health services for sex workers. Amsterdam: Correlation Network; 2008.

17. Vanwesenbeeck I. Another decade of social scientific work on sex work: a review of research 1990-2000. Annual Review of Sex Research. 2001;12(1):242-289.

18. Lazar C, Sanclemente C, Ferrer L, Folch C, Casabona J. Condom use among female sex workers in Catalonia: Why do they use a condom, why don 't they use it? AIDS Education and Prevention. 2015;27(2):180-193.

19. Murray L, Moreno L, Rosario S, Ellen J, Sweat $M$, Kerrigan D. The role of relationship intimacy in consistent condom use among female sex workers and their regular paying partners in the Dominican Republic. AIDS and Behavior. 2007;11:463-470.

20. Oliveira A. Da prostituição de apartamento na cidade de Lisboa: Características e significados. Porto: University of Porto; 2013. 
21. Pyett $P$, Haste B, Snow J. Risk practices for HIV infection and other STDs amongst female prostitutes working in legalized brothels. AIDS Care: Psychological and Socio-medical Aspects of AIDS/ HIV. 2010;8(1):85-94.

22. Parsons JT, Koken JA, Bimbi DS. The use of the Internet by gay and bisexual male escorts: sex workers as sex educators. AIDS Care. 2004;16(8):1021-1035.

23. Sanders T. Female sex workers as health educators with men who buy sex: Utilising narratives of rationalisations. Social Science \& Medicine. 2006;62:2434-2444.

24. O'Neill M, Barberet R. Victimization and the social organization of prostitution in England and Spain. In: Weitzer R, (ed.). Sex for sale: prostitution, pornography and the sex industry. New York: Routeledge; 2000. p. 123-137.

25. Pourette D. Les violences. In: Handman M-E, Mossuz-Lavau J, (dirs.). La prostitution à Paris. Paris: Éditions de La Martinière; 2005. p. 323-343.

26. Ribeiro M, Silva MC, Schouten J, Ribeiro FB, Sacramento O. Vidas na raia: Prostituição feminina em regiões de fronteira. Porto: Edições Afrontamento; 2008.

27. Costa AF. A pesquisa de terreno em Sociologia. In: Silva AS, Pinto JM, (orgs.). Metodologia das ciências sociais. Porto: Edições Afrontamento; 1986. p. 129-148.

28. Adler P. Wheeling \& dealing: An ethnography of an upper-level drug dealing and smuggling community. New York: Columbia University Press; 1993.

29. Oliveira A, Mota AM. Implementação e avaliação de um modelo de educação de pares para trabalhadores(as) do sexo: Relatório final da supervisão científica. Porto: University of Porto; 2012.
30. Oliveira A, Mota AM. Desenvolvimento e avaliação de um modelo de educação de pares para trabalhadores(as) do sexo: Relatório final da supervisão científica da $2^{\text {a }}$ fase do projeto. Porto: University of Porto; 2013.

31. Oliveira A. Social control of immigrant sex workers: transforming a group recognized as "at risk" into a group viewed as "a risk". International Journal of Migration, Health and Social Care. 2012;8(1):32-41.

32. Oliveira A. Prostituição de mulheres migrantes e saúde. In: Nogueira C, Magalhães S, (orgs.). Género e saúde: Novas (in)visibilidades. Porto: Edições Afrontamento; 2013. p. 75-85

33. Fernandes L. Figuras da vitimação colectiva. Revista Europeia de Inserção Social. 2006;1:57-78.

34. Oliveira A, Manita C. Prostituição, violência e vitimação. In: Gonçalves RA, Machado C, (coords.). Violência e vítimas de crimes. Coimbra: Quarteto; 2002.

35. Kurtz S, Surratt H, Iniciardi J, Kiley M. Sex work and "date" violence. Violence Against Women. 2004;10(4):357-385.

36. Fattah E. Understanding criminal victimization: An introduction to theoretical victimology. Scarborough: Prentice-Hall Canada Inc; 1991.

37. Goffman E. Stigmate: Les usages sociaux des handicaps. Paris: Les Editions de Minuit; 1963.

38. Smart L, Wegner D. Covering Up what can't be seen: Concealable stigma and mental control. Journal of Personality and Social Psychology. 1999;77(3):474-486.

39. Koken J, Bimbi D, Parsons J, Halkitis P. The experience of stigma in the lives of male internet escorts. Journal of Psychology \& Human Sexuality. 2004;16(1):13-32.

\section{FORMA DE CITAR}

Oliveira A, Fernandes L. Trabajadores del sexo y salud pública: intersecciones, vulnerabilidades y resistencia. Salud Colectiva. 2017;13(2):199-210. doi: 10.18294/sc.2017.1205.

Recibido: 31 de octubre de 2016 | Versión final: 18 de diciembre de 2016 | Aprobado: 2 de marzo de 2017

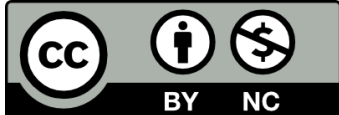

Este obra está bajo una licencia de Creative Commons Reconocimiento-NoComercial 4.0 Internacional. Reconocimiento - Permite copiar, distribuir y comunicar públicamente la obra. A cambio, se debe reconocer y citar al autor original. No Comercial - Esta obra no puede ser utilizada con finalidades comerciales, a menos que se obtenga el permiso. 